OPEN ACCESS

Edited by:

Sheue-yann Cheng,

National Cancer Institute (NCl),

United States

Reviewed by:

Sabine Costagliola

Fonds National de la Recherche

Scientifique (FNRS), Belgium

Risheng Ma,

Icahn School of Medicine at Mount

Sinai, United States

${ }^{*}$ Correspondence:

Qiliang Zhou

zhouql@med.niigata-u.ac.jp

${ }^{\dagger}$ Present address:

Yingchun Li,

Department of Hematology, Shengjing Hospital of China Medical University,

Shenyang, China

Specialty section:

This article was submitted to

Thyroid Endocrinology,

a section of the journal

Frontiers in Endocrinology

Received: 24 September 2020 Accepted: 10 November 2020 Published: 14 December 2020

Citation:

Ran Q, Zhou Q, Oda K, Yasue A, Abe $M, Y e X, L i Y$, Sasaoka $T$,

Sakimura K, Ajioka $Y$ and Saijo $Y$ (2020) Generation of Thyroid Tissues

From Embryonic Stem Cells via Blastocyst Complementation In Vivo.

Front. Endocrinol. 11:609697.

doi: 10.3389/fendo.2020.609697

\section{Generation of Thyroid Tissues From Embryonic Stem Cells via Blastocyst Complementation In Vivo}

\author{
Qingsong Ran ${ }^{1}$, Qiliang Zhou ${ }^{1 *}$, Kanako Oda ${ }^{2}$, Akihiro Yasue ${ }^{3}$, Manabu Abe ${ }^{4}, X_{\text {Xu }} \mathrm{Ye}^{1}$, \\ Yingchun $\mathrm{Li}^{1 \dagger}$, Toshikuni Sasaoka ${ }^{2}$, Kenji Sakimura ${ }^{4}$, Yoichi Ajioka ${ }^{5}$ and Yasuo Saijo ${ }^{1}$ \\ 1 Department of Medical Oncology, Niigata University Graduate School of Medical and Dental Sciences, Niigata, Japan, \\ ${ }^{2}$ Department of Comparative and Experimental Medicine, Brain Research Institute, Niigata University, Niigata, Japan, \\ ${ }^{3}$ Department of Orthodontics and Dentofacial Orthopedics, Institute of Biomedical Sciences, Tokushima University Graduate \\ School, Tokushima, Japan, ${ }^{4}$ Department of Animal Model Development, Brain Research Institute, Niigata University, Niigata, \\ Japan, ${ }^{5}$ Division of Molecular and Diagnostic Pathology, Niigata University Graduate School of Medical and Dental Sciences, \\ Niigata, Japan
}

The generation of mature, functional, thyroid follicular cells from pluripotent stem cells would potentially provide a therapeutic benefit for patients with hypothyroidism, but in vitro differentiation remains difficult. We earlier reported the in vivo generation of lung organs via blastocyst complementation in fibroblast growth factor 10 (Fgf10), compound, heterozygous mutant (Fgf10 Ex1 ${ }^{\text {mut }} / \mathrm{Ex}^{\mathrm{mut}}$ ) mice. Fgf10 also plays an essential role in thyroid development and branching morphogenesis, but any role thereof in thyroid organogenesis remains unclear. Here, we report that the thyroids of Fgf10 Ex1 ${ }^{\text {mut} /}$ Ex3 ${ }^{\text {mut }}$ mice exhibit severe hypoplasia, and we generate thyroid tissues from mouse embryonic stem cells (ESCs) in Fgf10 Ex1 ${ }^{\text {mut }} /$ Ex $3^{\text {mut }}$ mice via blastocyst complementation. The tissues were morphologically normal and physiologically functional. The thyroid follicular cells of $F g f 10$ Ex1 ${ }^{\text {mut }} /$ Ex $^{\text {mut }}$ chimeric mice were derived largely from GFP-positive mouse ESCs although the recipient cells were mixed. Thyroid generation in vivo via blastocyst complementation will aid functional thyroid regeneration.

Keywords: blastocyst complementation, embryonic stem cells, Fgf10, pluripotent stem cells, thyroid generation

\section{INTRODUCTION}

Continuous, oral thyroid hormone replacement therapy is indispensable for patients with hypothyroidism caused by total thyroidectomy or etiological factors. Although this is relatively simple, effective, safe, and inexpensive, it can be difficult to maintain the complex homeostatic interactions of various hormones $(1,2)$, and the side-effects of over-replacement include cardiac events and osteoporosis also cannot be ignored $(2,3)$. Regeneration and transplantation of thyroid tissue to physiologically supplement thyroid hormone levels is an alternative (radical) treatment strategy $(4,5)$. Derivation of thyroid follicular cells via directed differentiation of pluripotent stem cells (PSCs) in vitro, using growth factor-supplemented media, failed to regenerate mature thyroid follicular cells expressing the full genetic suite required for functional thyroid hormone biosynthesis (6-10). Using an embryonic stem cell (ESC) line hosting a GFP reporter-linked cDNA targeting the locus encoding the homeodomain-containing thyroid transcription factor 1 (TTF1 or Nkx2-1), Kurmann et al. reported the 
generation of functional thyrocytes via activation of bone morphogenetic protein (Bmp) and fibroblast growth factor (Fgf) signaling in vitro (11). Alternatively, transient forced overexpression of the transcription factors TTF1 and Paired box gene 8 (Pax8) of mouse or human ESCs allowed the cells to differentiate into functional thyroid follicular cells in vitro (12-15). However, the problems associated with in vitro generation of mature thyroid follicular tissue from PSCs, including low differentiation efficiency, the need for genetic labeling to sort and enrich progenitors, and the risk of tumor formation from undifferentiated PSCs after transplantation, limit the clinical applications of cell therapy.

Recently, in vivo models of organ generation via blastocyst complementation have shown promise. Generation of the pancreas $(16,17)$, kidney $(18,19)$, blood vasculature (20) and lung (21) via intra- or inter-species blastocyst complementation have been reported. Very recently, we used fibroblast growth factor 10, (Fgf10), compound, heterozygous mutant $\left(F g 10 \mathrm{Ex}^{\mathrm{mut}} / \mathrm{Ex} 3^{\mathrm{mut}}\right)$ mice to generate lungs via blastocyst complementation (22). Fgf10 $\mathrm{Ex}^{\mathrm{mut}} / \mathrm{Ex} 3^{\mathrm{mut}}$ mice exhibited limb and lung deficiencies, as did Fgf10 Ex1-/- and Fgf10 Ex3-/- mice, as well as other Fgf10knockout mice (23-25). Complementation with ESCs enabled Fgf10 $\mathrm{Ex} 1^{\mathrm{mut}} / \mathrm{Ex} 3^{\mathrm{mut}}$ mice to survive to adulthood without any abnormality.

In contrast to the relatively distinct role played by Fgf10 in lung development and branching morphogenesis (23, 24, 26-28), indefiniteness remains in thyroid organogenesis. Thyroid agenesis has been reported in mice deficient in Fgf10 (24) or its receptor Fgfr2b (29), indicating that Fgf10-Fgfr2b signaling plays a crucial role in thyroid organogenesis. However, although the thyroid primordium was absent at E13, the stage at which thyroid morphogenesis was impaired was not explored. Nkx2$1^{+} /$Sox $9^{+}$thyroid progenitors were detected in the thyroid placode at E9.5; weak expression of Fgfr2b in the thyroid primordium at E12.5; and distinct expression of Fgf10 in the mesenchyme at E15.5 (30). By contrast, it has been reported that Fgf10-null mutant mouse embryos did not exhibit thyroid agenesis but rather severe hypoplasia (the thyroid was shaped normally) (30, 31). Similarly, conditional knockout of Fgf10 (Wnt1cre Fgf10 fl/fl) in neural crest, from which several head tissues are derived (including the mesenchyme around the developing thyroid glands), resulted thyroid remnants (31). Therefore, we explored the thyroid phenotype of Fgf10 Ex1 ${ }^{\text {mut }}$ $\mathrm{Ex} 3^{\mathrm{mut}}$ mice and the possibility of thyroid generation in such mice from PSCs (thus via blastocyst complementation).

Here, we report that the thyroids of Fgf10 Ex1 ${ }^{\mathrm{mut}} / \mathrm{Ex} 3^{\text {mut }}$ mice are normally shaped but severely hypoplastic. Complementation with ESCs rescued thyroid organogenesis. Generation of thyroids in vivo via blastocyst complementation will aid functional thyroid regeneration.

\section{MATERIALS AND METHODS}

\section{Generation of Fgf10 Ex1 ${ }^{\text {mut }} / \mathrm{Ex} 3^{\text {mut }}$ Mice and Chimeric Mice}

All animal experiments were approved by the Institutional Animal Care and Use Committee of Niigata University, Niigata,
Japan (approval number SA00233). Fgf10 Ex1 ${ }^{\text {wild/mut }}$ and Fgf10 $\mathrm{Ex} 3^{\text {wild/mut }}$ mice were generated using the CRISPR/Cas9 system as described in our previous report (22). Fgf10 $\mathrm{Ex} 1^{\mathrm{mut}} / \mathrm{Ex} 3^{\mathrm{mut}}$ mice were obtained by intercrossing Fgf10 Ex1 ${ }^{\text {wild/mut }}$ mice with Fgf10 $\mathrm{Ex} 3^{\text {wild/mut }}$ mice. Generation of $\mathrm{Fg} f 10 \mathrm{Ex} 1^{\text {mut }} / \mathrm{Ex} 3^{\text {mut }}$ chimeric mice via blastocyst complementation proceeded as described previously (22). Briefly, embryos were prepared via in vitro fertilization of Fgf10 Ex3-/+ ova with Fgf10 Ex1-/+ sperm, and five to eight GFPexpressing mouse RENKA C57BL/6NCrlCrlj ESCs (\#CFSEGFP27; Brain Research Institute, Niigata University) were prepared and microinjected into the perivitelline space of eightcell/morula-stage embryos. After further culture in vitro, the embryos were transferred into the uteri of pseudopregnant, recipient ICR female mice. Genotyping of the Fgf10 Ex1 ${ }^{\mathrm{mut} /}$ Ex $3^{\text {mut }}$ mice and chimeric mice were performed using the Surveyor System and DNA sequencing, as described previously (22).

\section{Histological Analysis}

Mouse tissues were fixed in $10 \%(\mathrm{v} / \mathrm{v})$ neutral buffered formalin, embedded in paraffin, sectioned, and the sections deparaffinized with xylene and hydrated in a graded series of ethanol baths. Hematoxylin and eosin (H\&E) and immunofluorescence staining were performed as described previously (22). The primary antibodies were anti-GFP polyclonal antibody (goat IgG, 1:200; \#GTX26673; GeneTex, Irvine, CA, USA); antiTTF1 monoclonal antibody (rabbit IgG, 1:200; \#ab76013; Abcam, Cambridge, UK); anti-FOXE1 polyclonal antibody (rabbit IgG, 1:200; \#bs-0446r; Bioss, Woburn, MA, USA); antiPax8 antibody (rabbit IgG, 1:200; \#10337-1-AP; Proteintech, Chicago, IL, USA); anti-thyroglobulin monoclonal antibody (rabbit IgG,1:200; \#ab156008; Abcam); anti-T3 polyclonal antibody (rabbit IgG, 1:200; \#MBS2001953; MyBioSource, San Diego, CA, USA); anti-calcitonin polyclonal antibody (rabbit IgG, 1:200; \#GTX134005; GeneTex); anti-vimentin monoclonal antibody (rabbit IgG, 1:200; \#ab92574; Abcam); and anti-Ki-67 polyclonal antibody (rabbit IgG, 1:200; \#ab15580, Abcam). Donkey anti-goat IgG-Alexa Fluor 488 (1:200; \#A11055; Invitrogen, Carlsbad, CA, USA) and donkey anti-rabbit IgGAlexa Fluor 594 (1:200; \#A21207; Invitrogen) served as secondary antibodies. Nuclei were counterstained with 4',6diamidino-2-phenylindole (DAPI) and fluorescence images acquired using a C1si confocal microscope (Nikon, Tokyo, Japan).

TTF1-positive cells were counted in over 1,500 cells in at least three images (200× magnification) randomly selected from the thyroids of each mouse. GFP-positive cells among TTF1-positive cells were counted and the percentage of GFP/TTF1-positive cells was then calculated.

\section{Contrast-Enhanced Micro-Computed Tomography}

To explore the macroscopic phenotypes of the thyroid tissues of Fgf10 Ex1 ${ }^{\text {mut }} / \mathrm{Ex}^{\text {mut }}{ }^{\text {neonatal mice }}$ and $F g f 10 \mathrm{Ex} 1^{\mathrm{mut}} / \mathrm{Ex} 3^{\mathrm{mut}}$ chimeric neonatal mice, contrast-enhanced micro-CT analysis was performed as described previously (22) with slight modifications. Briefly, neonatal mice were first fixed in 
$4 \%(\mathrm{v} / \mathrm{v})$ paraformaldehyde at $4^{\circ} \mathrm{C}$ for 2 days. A midline cervical incision was then created and the larynx, trachea, and thyroid exposed. Then, the mice were immersed in 25\% (v/v) Lugol's iodine solution at room temperature for 5 days. Subsequently, the samples were scanned using a micro-CT device (Nittetsu Elex, Tokyo, Japan) and the data analyzed with the aid of TRI/ 3D-Bon software (Ratoc System Engineering Co. Ltd., Tokyo, Japan).

\section{Enzyme-Linked Immunosorbent Assays}

Serum tri-iodothyronine (T3) and thyroxine (T4) concentrations were measured using ELISA kits (CSB-E05086m for T3, CSBE05083m for T4; CUSABIO, Wuhan, China), according to the manufacturer's protocols. Briefly, $50 \mu \mathrm{l}$ of standards or blood samples was added to 96 -well plates, followed by $50 \mu \mathrm{l}$ of conjugate reagents; incubation proceeded for $60 \mathrm{~min}$ at $37^{\circ} \mathrm{C}$. The liquid was aspirated, the wells washed three times, $50 \mu \mathrm{l}$ of the HRP-avidin reagent added, and the plates incubated for $30 \mathrm{~min}$ at $37^{\circ} \mathrm{C}$. The liquid was aspirated, the wells washed three times, and $50 \mu \mathrm{l}$ of substrates $\mathrm{A}$ and B added. After incubation for $15 \mathrm{~min}$ at $37^{\circ} \mathrm{C}$ in the dark, $50 \mu \mathrm{l}$ of stop solution was added and the optical density at $450 \mathrm{~nm}$ measured within 10 min using a microplate reader. All tests were performed in duplicate.

\section{Statistical Analysis}

Data are presented as the means \pm standard deviations. One-way analysis of variance and the Tukey-Kramer test were used to assess the significance of differences. A $p$-value $<0.05$ was deemed to indicate significance.

\section{RESULTS}

\section{Fgf10 Ex1 ${ }^{\text {mut }} /$ Ex $^{\text {mut }}$ Mice Exhibit Severe Thyroid Hypoplasia}

Fof10 $\mathrm{Ex} 1^{\mathrm{mut}} / \mathrm{Ex} 3^{\mathrm{mut}}$ mice were generated as previously reported (22). Consistent with the data of a recent study on embryonic growth of the thyroid gland in Fgf10-null mutant mice (30), neonatal Fgf10 $\mathrm{Ex}^{\mathrm{mut}} / \mathrm{Ex}^{\mathrm{mut}}$ mice exhibited bilateral thyroid remnants (Figure 1A) on micro-CT analysis. Serial sections of the entire glands $(n=5)$ confirmed that the thyroids were normally shaped but smaller than those of $\mathrm{Fgfl} \mathrm{O}^{\text {wild }} /$ wild neonates (Supplemental Videos 1 and 2). H\&E and immunofluorescence staining indicated that the hypoplastic thyroids glands of Fgf10 $\mathrm{Ex} 1^{\mathrm{mut}} / \mathrm{Ex} 3^{\mathrm{mut}}$ mice had a lower proportion of parenchyma, decreased branching, and fewer follicles than normal mouse thyroids (Figures 1B, C). Immunofluorescence staining indicated that the number of thyroid cells expressing TTF1 and Pax8 (the most important transcription factors in terms of thyroid gland organogenesis) was decreased in neonatal $\mathrm{FgflO} \mathrm{Ex}^{\mathrm{mut}} / \mathrm{Ex} 3^{\mathrm{mut}}$ mice compared to neonatal $\mathrm{Fg} f 10^{\text {wild }} /$ wild mice (Figure 1C). Although the protein levels seem to be similar, the total expression levels of thyroglobulin ( $\mathrm{Tg}$ ) (a precursor protein of thyroid hormone) and tri-iodothyronine (T3) were reduced in neonatal $\mathrm{FgflO} \mathrm{Ex}^{\mathrm{mut}} / \mathrm{Ex} 3^{\text {mut }}$ mice (Figure 1C). Ki-67 positive proliferating cells were obviously reduced in thyroids of neonatal
Fgf10 Ex1 ${ }^{\mathrm{mut}} / \mathrm{Ex}^{\mathrm{mut}}$ mice compared to neonatal $\mathrm{Fg} 10^{\text {wild }} /$ wild mice (Figure 1C). The expression of calcitonin in the neonatal Fgflo $\mathrm{Ex} 1^{\mathrm{mut}} / \mathrm{Ex} 3^{\text {mut }}$ mice did not seem to decrease significantly (Figure 1C), in agreement with a previous report that Fgf10 is not involved in parafollicular cell differentiation (30).

\section{Generation of Thyroid Tissues in Fgf10 Ex1 $1^{\text {mut }} /$ Ex3 $^{\text {mut }}$ Mice}

We next sought to generate thyroid tissues from PSCs in Fgflo $\mathrm{Ex} 1^{\mathrm{mut}} / \mathrm{Ex} 3^{\mathrm{mut}}$ mice via blastocyst complementation. Micro-CT confirmed the existence of thyroids adjacent to the trachea at the front of the neck of neonatal Fgf10 $\mathrm{Ex} 1^{\mathrm{mut}} / \mathrm{Ex} 3^{\text {mut }}$ chimeras; the glands were of normal shape and size (Figure 1A). The thyroids of Fgf10 $\mathrm{Ex} 1^{\mathrm{mut}} / \mathrm{Ex} 3^{\mathrm{mut}}$ chimeric neonates (Figure $\mathbf{1 B}$ and Supplemental Video 3) were histologically normal (thus similar to those of $\mathrm{Fg} f 10^{\text {wild }} /$ wild neonates) (Figure 1B and Supplemental Video 1). The thyroid tissues of $\mathrm{Fg} 10 \mathrm{Ex} 1^{\mathrm{mut}} / \mathrm{Ex} 3^{\text {mut }}$ chimeras exhibited high-level GFP expression compared to those of Fgf10 wild/ wild neonates (Figures 1D, E), indicating a major contribution from GFP-expressing mouse ESCs. The levels of TTF1, Tg, and T3 in the thyroids of neonatal Fgf10 Ex1 ${ }^{\text {mut }} / \mathrm{Ex} 3^{\text {mut }}$ chimeras (Figure 1F) were similar to those of neonatal Fgf $10^{\text {wild }} /$ wild mice (Figure 1C). The GFP expression of TTF1positive follicular cells predominated but was mosaic, while those of calcitonin-positive parafollicular cells and vimentin-positive stromal cells showed no preponderance (Figure 1F). These data indicated that thyroid tissues were generated in $\mathrm{FgflO} \mathrm{Exl}^{\mathrm{mut} /}$ $\mathrm{Ex} 3^{\text {mut }}$ mice via blastocyst complementation.

\section{Characterization of the Thyroids of Adult Fgf10 Ex1 ${ }^{\text {mut }} / \mathrm{Ex}^{\mathrm{mut}}$ Chimeric Mice}

We showed that survival of $F g f 10 \mathrm{Ex} 1^{\mathrm{mut}} / \mathrm{Ex} 3^{\mathrm{mut}}$ mice to adulthood was rescued by complementation with mouse ESCs (22). Next, we analyzed the thyroid tissues of five Fgf10 Ex1 ${ }^{\mathrm{mut} /}$ $\mathrm{Ex} 3^{\text {mut }}$ adult chimeric mice. We lacked data on adult Fgf10 $\mathrm{Ex} 1^{\mathrm{mut}} / \mathrm{Ex} 3^{\text {mut }}$ mice because they died immediately after birth; they had no lungs. The low proportion of parenchyma in the thyroids of $\mathrm{Fgf10} \mathrm{Ex}^{\mathrm{mut}} / \mathrm{Ex} 3^{\mathrm{mut}}$ neonates (Figure 1B) recovered in the thyroid tissues of adult $\mathrm{Fgf10} \mathrm{Ex} 1^{\mathrm{mut}} / \mathrm{Ex} 3^{\text {mut }}$ chimeras (Figure 2A). The thyroid follicles of adult $\mathrm{Fg} 10 \mathrm{Ex} 1^{\mathrm{mut}} / \mathrm{Ex} 3^{\mathrm{mut}}$ chimeras were well-organized spheres lined with follicular cells surrounding the lumina that contained a colloid, as in adult Fof $10^{\text {wild }} /$ wild mice (Figure 2A). The thyroid follicular cells of adult Fgf10 $\mathrm{Ex}^{\mathrm{mut}} / \mathrm{Ex} 3^{\text {mut }}$ chimeras expressed TTF1, FOXE1 (formerly TTF2), and Pax8 at levels similar to those of adult Fgf $10^{\text {wild }} /$ wild mice (Figure 2B). Calcitonin-positive parafollicular cells were detected in connective tissue adjacent to the thyroid follicles, as in adult $\mathrm{Fg} f 10^{\text {wild }} /$ wild mice (Figure 2B). Thus, the

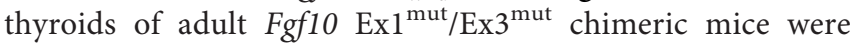
histologically normal.

Next, we investigated the contribution of GFP-expressing mouse ESCs to the thyroids. Extremely strong, diffuse, GFP expression across all thyroid tissues was observed in Fgf10 $\mathrm{Ex} 1^{\mathrm{mut}} / \mathrm{Ex} 3^{\mathrm{mut}}$ adult chimeras compared to adult $\mathrm{Fg} f 10^{\text {wild }} /$ wild mice or $F g f 10 \mathrm{Ex} 1^{\text {wild }} / \mathrm{Ex} 3^{\text {mut }}$ chimeras (Figure 2B). In Fgf10 $\mathrm{Ex} 1^{\mathrm{mut}} / \mathrm{Ex} 3^{\mathrm{mut}}$ adult chimeric mice, large proportions of the 

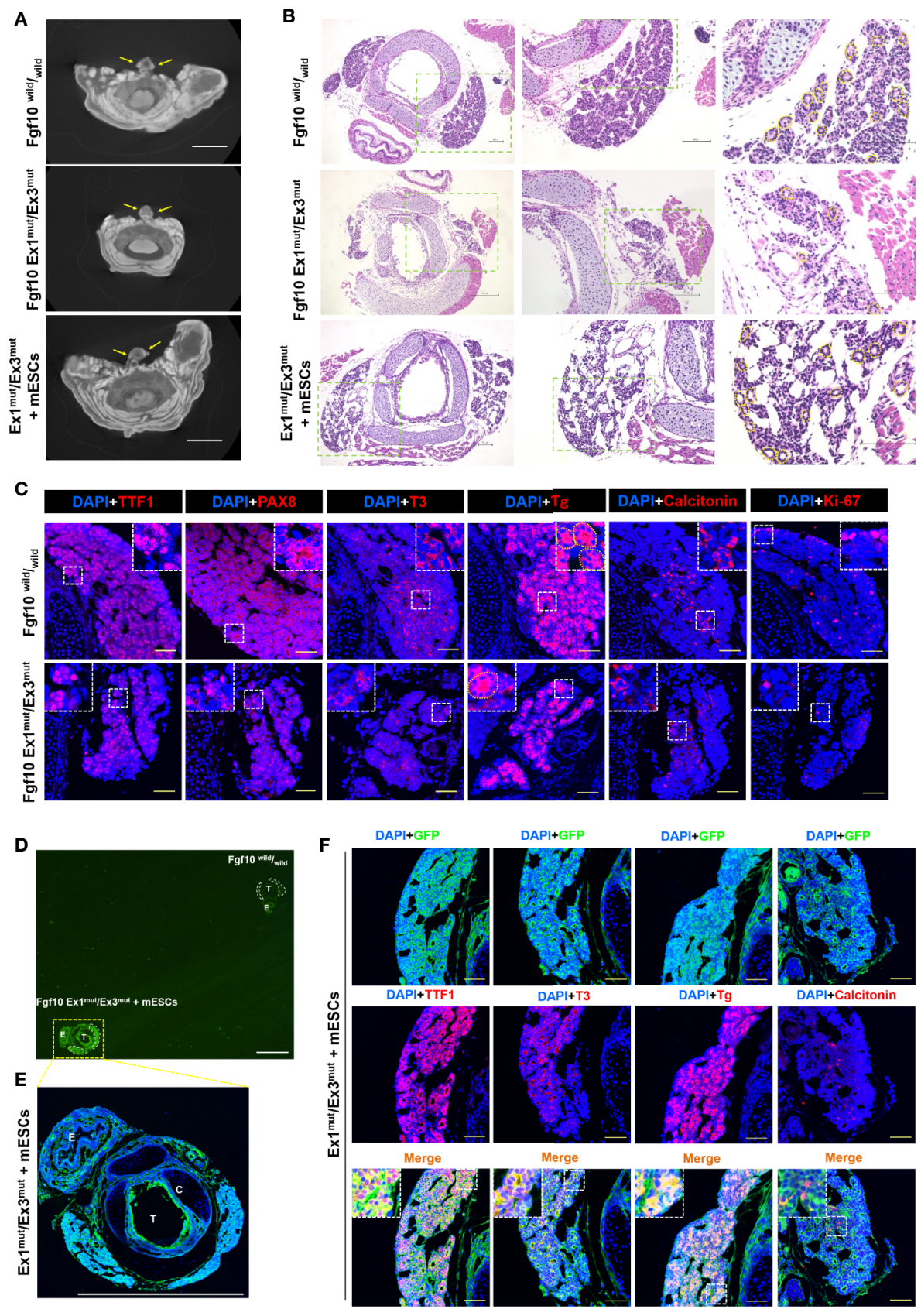

DAPI+GFP

DAPI+GFP

FIGURE 1 Characterization of the thyroids of Fgf10 Ex1 ${ }^{\text {mut }} /$ Ex $^{\text {mut }}$ neonates and Ex1 ${ }^{\text {mut }} /$ Ex $3^{\text {mut }}$ chimeric neonates complemented with mouse embryonic stem

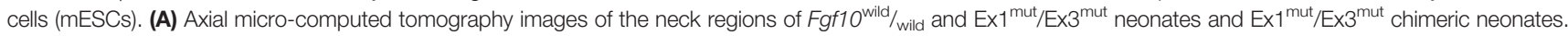
Yellow arrows indicate thyroid lobes adjacent to the tracheae. Scale bar $=2 \mathrm{~mm}$. (B) Hematoxylin and eosin staining of cervical cross-sections of Fgf10 wild/wild and

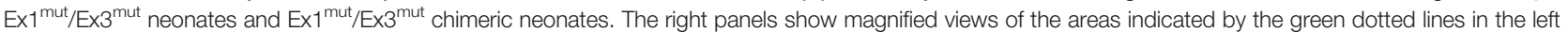
panels. Scale bars $=100 \mu \mathrm{m}$. (C) Immunofluorescence staining of the thyroids of Fgf10 wild/wild and Ex1 mut/Ex3 ${ }^{\text {mut }}$ neonates for various markers (red): TTF1, thyroid transcription factor1; PAX8, paired box gene 8; T3, tri-iodothyronine; Tg, thyroglobulin; Calcitonin and Ki-67. Nuclei were stained with DAPI (blue). Scale bars = 50 $\mu \mathrm{m}$. Yellow dotted lines in (B, C) indicated representative thyroid follicles. (D-F) Immunofluorescence staining of the thyroid of an Fgf10 Ex1 ${ }^{\text {mut }} /$ Ex3 $^{\text {mut }}$ chimeric neonate. (D) A low magnification image acquired using a stereo fluorescence microscope. The thyroid of an Fgf10wild/wild neonate placed on the same slide served as the control. White dotted lines indicate the thyroid glands. (E) Image acquired using a confocal microscope (with slight magnification) of the tissue indicated by the yellow dotted box in (D). Scale bars $=1 \mathrm{~mm}$. T, trachea; C, cartilage; E, esophagus. (F) Immunofluorescence staining of the thyroids of Fgf10 Ex1 ${ }^{\text {mut }} / \mathrm{Ex}^{\text {mut }}$ neonates for GFP (green) and thyroid markers (red): TTF1, T3, Tg and Calcitonin. Nuclei were stained with DAPI (blue). Insets in (C, E) show magnified views of the areas indicated with white dotted lines. Scale bars $=50 \mu \mathrm{m}$. 
A

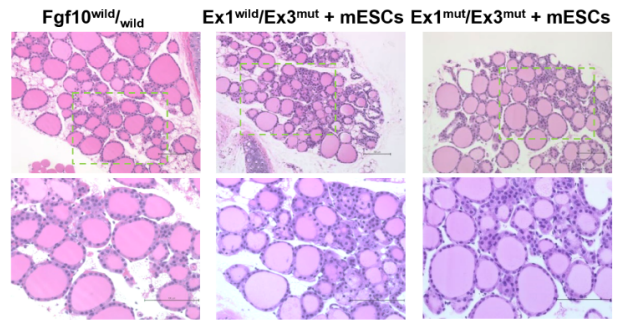

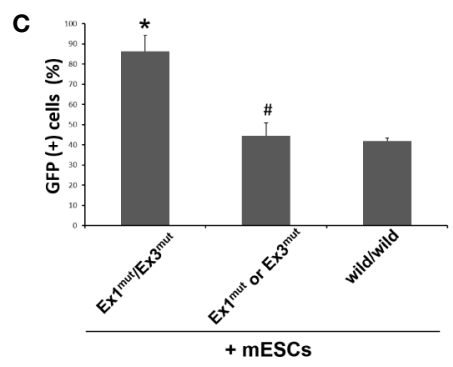

B

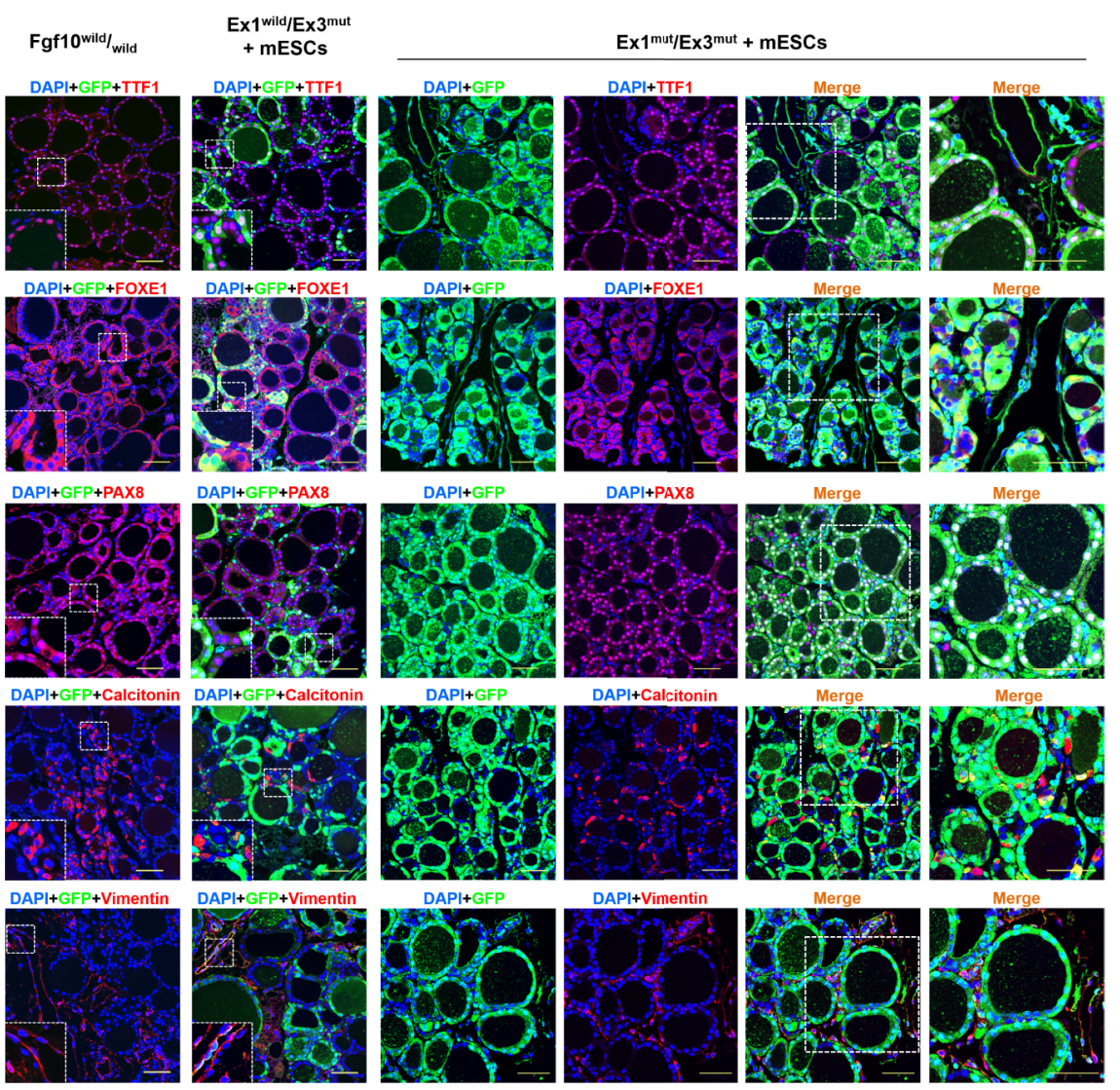

FIGURE 2 | Characterization of the thyroids of adult Fgf10 Ex1 ${ }^{\text {mut }} /$ Ex3 ${ }^{\text {mut }}$ chimeric mice complemented with mouse embryonic stem cells (mESCs). (A) Hematoxylin and eosin staining of thyroid tissues from adult Fgf10 wild/wild mice, and Fgf10 Ex1 ${ }^{\text {wild }} /$ Ex $3^{\text {mut }}$ and Ex1 ${ }^{\text {mut }} /$ Ex $3^{\text {mut }}$ chimeric mice. The bottom panels show magnified

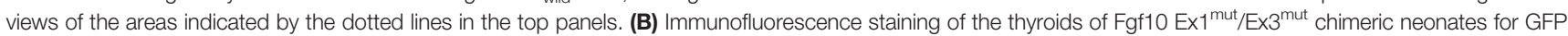
(green) and various markers (red): TTF1, thyroid transcription factor 1; FOXE1, forkhead box E1; and PAX8, paired box gene 8 for follicular cells, Calcitonin for parafollicular cells, and Vimentin for stromal cells. Nuclei were stained with DAPI (blue). Fgf10 wild/wild and Fgf10 Ex1 wild/Ex3 ${ }^{\text {mut }}$ chimeric mice served as controls. Insets show magnified views of the areas indicated with white dotted lines. The right panels show magnified views of the areas indicated by the dotted lines in the left panels. Scale bars $=100 \mu \mathrm{m}$ for (A); $50 \mu \mathrm{m}$ for (B). (C) Enumeration of GFP/TTF1-positive thyroid follicular cells in adult Fgf10 Ex1 ${ }^{\text {mut }} /$ Ex3 $^{\text {mut }}$, Fgf10 Ex1 ${ }^{\text {mut }}$ or $\mathrm{Ex}^{\mathrm{mut}}$, and Fgf10 ${ }^{\text {wild }} /$ wild chimeric mice. Data are expressed as the means \pm standard deviations; $\mathrm{n}=3 /$ group. ${ }^{*} p<0.05$ versus other treatments; ${ }^{*} p>0.05$ versus Fgf10 wild/wild chimeras.

TTF1-, FOXE1-, and Pax8-positive follicular cells were GFPpositive, indicating that the cells were derived principally from mouse ESCs (Figure 2B). The extent of GFP expression in nonfollicular regions, including parafollicular cells, blood vessels, and connective tissues, did not differ between the Fgflo Ex1 ${ }^{\mathrm{mut}} /$ $\mathrm{Ex} 3^{\text {mut }}$ and $\mathrm{Ex} 1^{\text {wild }} / \mathrm{Ex} 3^{\text {mut }}$ chimeras (Figure 2B). Moreover, $86.4 \pm 7.9 \%$ of follicular cells in adult Fgf10 Ex1 $1^{\text {mut }} / \mathrm{Ex} 3^{\text {mut }}$ chimeras were derived from GFP-positive mouse ESCs, a greater proportion than in adult $\mathrm{Fg} f 10^{\text {wild }} /$ wild and $\mathrm{Ex} 1^{\text {mut }}$ or Ex $3^{\text {mut }}$ chimeras (Figure $2 \mathrm{C}$ ). Next, we assessed the physiological function of the thyroid tissues of adult Fgf10 $\mathrm{Ex} 1^{\mathrm{mut}} / \mathrm{Ex} 3^{\text {mut }}$ chimeras. Immunofluorescence staining confirmed cytosolic expression of $\mathrm{Tg}$ and deposition thereof in the thyroid follicular lumina (Figure 3A). T3 was also detected in 

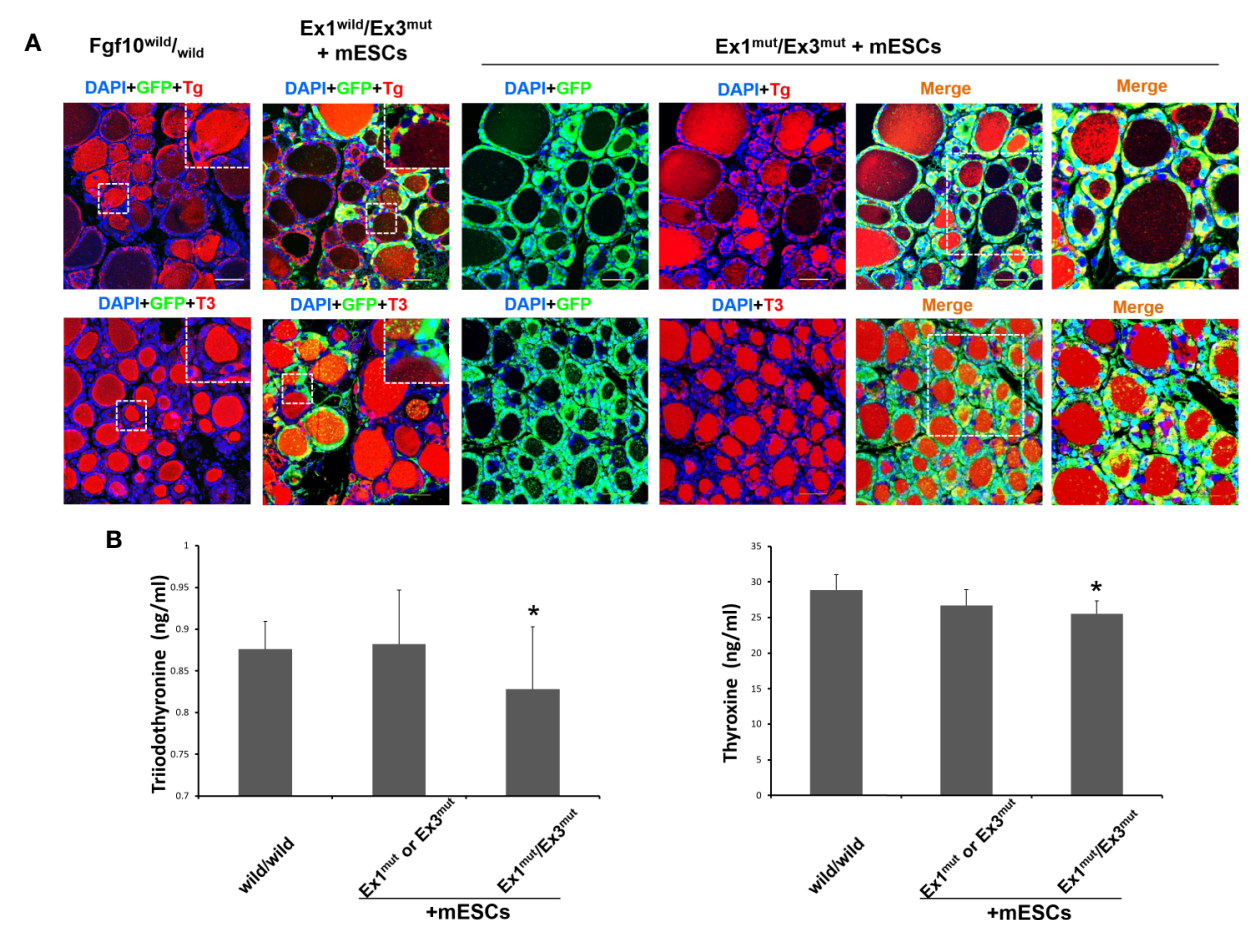

FIGURE 3 | In vivo thyroid functionality assessment in adult Fgf10 Ex1 ${ }^{\text {mut }} /$ Ex3 ${ }^{\text {mut }}$ chimeric mice complemented with mouse embryonic stem cells (mESCs). (A) Immunofluorescence staining of the thyroids. Thyroid follicles were analyzed by staining for GFP (green) and markers of thyroid function. Tg, thyroglobulin; T3, triiodothyronine. Insets show magnified views of the areas indicated with white dotted lines. The right panels show magnified views of the areas indicated by dotted lines in the left panels. Nuclei were stained with DAPI (blue). Fgf10 wild/wild and Fgf10 Ex1 wild/Ex3 ${ }^{\text {mut }}$ chimeric mice served as controls. Scale bar $=50 \mu$ m. (B) ELISA analyses of serum tri-iodothyronine (T3) and thyroxine (T4) concentrations in adult Fgf10 Ex1 ${ }^{\text {mut }} /$ Ex3 ${ }^{\text {mut }}$ chimeric mice. Adult Fgf10 ${ }^{\text {wild }} /$ wild $_{\text {and Fgf10 Ex1 }}$ mut or Ex $^{\text {mut }}$

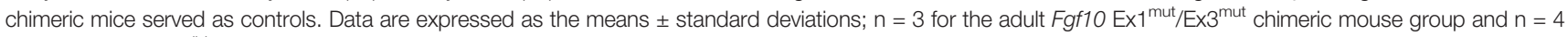
for the adult Fgf10 ${ }^{\text {wild }} /$ wild and Fgf10 Ex1 ${ }^{\text {mut }}$ or Ex3 ${ }^{\text {mut }}$ chimeric mouse groups; ${ }^{\star} p>0.05$ versus other treatments.

the colloid, as in adult $F g f 10^{\text {wild }} /$ wild mice (Figure 3A). ELISA confirmed that the plasma T3 and thyroxine (T4) levels of adult $\mathrm{Fg} 10 \mathrm{Ex} 1^{\mathrm{mut}} / \mathrm{Ex} 3^{\mathrm{mut}}$ chimeras were similar to those of adult Fgf $10^{\text {wild }} /$ wild mice and $\mathrm{Ex} 1^{\text {mut }}$ or $\mathrm{Ex} 3^{\text {mut }}$ chimeric mice (Figure 3B). Thus, thyroids of adult $\mathrm{Fg} f 10 \mathrm{Ex} 1^{\mathrm{mut}} / \mathrm{Ex} 3^{\text {mut }}$ chimeras were functional. Thus, the functional thyroid follicles were generated principally from mouse ESCs in adult Fgf10 $\mathrm{Ex} 1^{\mathrm{mut}} / \mathrm{Ex} 3^{\mathrm{mut}}$ chimeric mice, via blastocyst complementation.

\section{DISCUSSION}

We generated thyroid tissues in $\mathrm{Fg} f 10 \mathrm{Ex} 1^{\mathrm{mut}} / \mathrm{Ex} 3^{\text {mut }}$ mice with severely hypoplastic thyroids via blastocyst complementation with mouse ESCs. The generated thyroids were morphologically normal and physiologically functional compared to those of Fgf $10^{\text {wild }} /$ wild mice. The generated thyroid tissues exhibited significant contributions from GFP-positive ESCs but the recipient cells were mixed.

Early during mouse thyroid development, thyroid progenitors expressing a specific combination of four critical transcription factors [Nkx2-1, Pax8, FOXE1 (Forkhead Box E1), and HHEX (hematopoietically expressed homeobox)] assemble to form the thyroid bud in the anterior foregut endoderm (32, 33). These transcription factors are linked to an integrated regulatory network that controls thyroid survival and migration during organogenesis, via cell-autonomous mechanisms (32, 33). Deletion of a gene encoding any of these transcription factors triggers athyreosis or severe thyroid hypoplasia (34). Fgf10 plays essential roles in the development of many organs such as the thyroid, limbs, lungs, and pituitary and salivary glands, mediated principally via the mesenchymal-epithelial interaction signaled through the receptor Fgfr2-IIIb $(24,35)$. Mice deficient in Fgf10 or Fgfr2b exhibit athyreosis, indicating that Fgf10 is required for thyroid budding and branching morphogenesis $(24,29)$. However, a recent study reported that most Fgf10-null mouse embryos exhibited small, unilateral remnant thyroids, indicating that organogenesis proceeded even in the complete absence of Fgf10 (31). Conditional, neural crest Fgf10 knock-out reduced thyroid size to a lesser degree than in the null mutant, suggesting that a source of Fgf10 apart from the neural crest might be available to assist thyroid development (31). A recent work on thyroid branching morphogenesis showed that normally shaped, symmetrical thyroids were present in Fgf10-null mutant mouse embryos, but were severely hypoplastic (30). Fgf10-Fgfr2b signaling may thus be dispensable in terms of thyroid 
specification and early development, but is required to regulate organogenesis (30). We found that the thyroids of neonatal, Fgf10 compound heterozygous mutant $\left(F g f 10 \mathrm{Ex}^{\mathrm{mut}} / \mathrm{Ex}^{\mathrm{mut}}\right)$ mice were severely hypoplastic but symmetrically residual, supporting the above observations in mouse embryos $(30,31)$. Furthermore, complementation with Fgf10wild-type ESCs rescued thyroid organogenesis both histologically and functionally in Fgf10 $\mathrm{Ex} 1^{\mathrm{mut}} / \mathrm{Ex} 3^{\mathrm{mut}}$ mice, indicating that Fgf10 played essential roles in late thyroid development and organogenesis.

Although Fgf10 seems to be dispensable in terms of thyroid specification and early thyroid development, Fgf10-induced branching growth has been reported to account for over $80 \%$ of thyroid enlargement before birth (30). Given the symmetrical, severe thyroid hypoplasia of $\mathrm{Fg} f 10 \mathrm{Ex} 1^{\mathrm{mut}} / \mathrm{Ex} 3^{\mathrm{mut}}$ mice, we expected that it might be possible to generate functional thyroid tissues from PSCs in such mice via blastocyst complementation. Indeed, high proportions of the thyroid follicular cells of Fgflo $\mathrm{Ex} 1^{\mathrm{mut}} / \mathrm{Ex} 3^{\mathrm{mut}}$ adult chimeric mice were GFP-positive (Figure 2B), indicating major contributions from donor ESCs. Localized Fgf10 expression by donor ESCs in the mesenchyme around developing thyroid glands would act non-selectively (via Fgfr2IIIb-mediated mesenchymal-epithelial interaction signaling) on both GFP-positive donor cells ( $F g f 10^{\text {wild }} /$ wild $)$ and GFP-negative host cells (Fgf10 $\left.\mathrm{Ex} 1^{\mathrm{mut}} / \mathrm{Ex} 3^{\mathrm{mut}}\right)$ resident in the endoderm. However, other mechanisms [such as ectopic expression of Fgf10 in the GFP-positive donor epithelium ( $F g f 10^{\text {wild }} /$ wild $)$, as indicated during lung generation via blastocyst complementation] (22) may explain in the relative preponderance of GFP-positive donor ESCs during thyroid development compared to the level in the Fgf10 $\mathrm{Ex}^{\mathrm{mut}} / \mathrm{Ex} 3^{\mathrm{mut}}$ host epithelium. Importantly, ESCderived thyroid follicles expressed and deposited T3 as did adult Fof $10^{\text {wild }} /$ wild mice (Figure 3A). These data, together with the ELISA results indicating that adult $F g f 10 \mathrm{Ex} 1^{\text {mut }} / \mathrm{Ex} 3^{\text {mut }}$ chimeras had normal T3 and T4 plasma levels compared to adult Fgf10 $10^{\text {wild }}$ wild mice (Figure 3B), indicated that the mature, functional thyroid follicle tissues of adult $\mathrm{Fg} f 10 \mathrm{Ex} 1^{\mathrm{mut}} / \mathrm{Ex} 3^{\text {mut }}$ chimeras were generated predominantly from ESCs.

Directed in vitro differentiation of PSCs using growth factors has been reported, but failed to regenerate mature thyroid follicular cells (6-10). Derivation of functional thyroid follicular cells in vitro from mouse and human induced PSCs $(4,11)$, mouse ESCs $(12,13,15)$, and human ESCs (14) has been reported using several protocols. However, the generation of such cells from PSCs is inefficient; enrichment and sorting of precursor cells currently requires genetic editing (TTF1 and Pax8 overexpression or labeling of targeted alleles) $(34,36)$. Also, the risk of tumor formation from undifferentiated PSCs on transplantation after in vitro differentiation cannot be ignored. Our current work indicates that mature, functional thyroid follicular cells can be generated from PSCs via blastocyst complementation. Although the generated thyroid tissues in $\mathrm{Fg} 10 \mathrm{Ex} 1^{\mathrm{mut}} / \mathrm{Ex} 3^{\text {mut }}$ chimeras were mixtures of donor and host cells, this is not an argument against thyroid regeneration, because transplantation of mature thyroid follicular cells (not the organ) would suffice as therapy for patients with hypothyroidism. Sorting of PSC-derived mature follicular cells or follicular tissues is required. Furthermore, the low efficiency of adult $\mathrm{Fg} f 10 \mathrm{Ex} 1^{\mathrm{mut}} / \mathrm{Ex} 3^{\text {mut }}$ chimera generation (5 adult compound heterozygous chimeras weaned from 76 neonatal chimeras obtained by transplantation of 638 blastocysts) (22) and the undesirable thyroid chimerism of the present study require attention. The use of a conditional knockout method or other knockout targets such as Nkx2-1, Pax8, or Fgf2 (all of which are essential for early thyroid development) might be useful. Wen et al. recently generated lung and thyroid epithelial cell lineages almost entirely from mouse ESCs in Nkx2-1 knockout mice via blastocyst complementation (37). Exploring the possibility of generation of PSC-derived thyroid tissues via inter-species blastocyst complementation in rodents or livestock remains to be investigated (22). Another concern is that human PSCs-derived cells will appear in the brains and gonads of livestock, especially when generating human organs from PSCs in livestock using the current inter-species blastocyst complementation technique. The use of committed stem or progenitor cells, or PSCs genetically modified to restrict their differentiation potential, would address this issue (16), but clinical application remains some way off.

In summary, we showed that Fgf10 played an essential role in thyroid development and that thyroid tissues generated in thyroid hypoplastic $\mathrm{Fg} 10 \mathrm{Ex} 1^{\mathrm{mut}} / \mathrm{Ex} 3^{\mathrm{mut}}$ mice were largely derived from mouse ESCs via blastocyst complementation. Generation of PSCderived thyroid tissues via blastocyst complementation is a promising approach to thyroid regeneration.

\section{DATA AVAILABILITY STATEMENT}

The original contributions presented in the study are included in the article/Supplementary Material. Further inquiries can be directed to the corresponding author.

\section{ETHICS STATEMENT}

The animal study was reviewed and approved by Institutional Animal Care and Use Committee of Niigata University.

\section{AUTHOR CONTRIBUTIONS}

QR performed experiments, contributed to data analysis and interpretation, and assisted with manuscript preparation. $\mathrm{KO}$ and TS performed the embryo manipulation and animal experiments, and contributed to the analysis and interpretation of mouse data. AY generated the Fgflo knockout mouse and contributed to DNA analysis. MA and KS prepared the GFPpositive mouse ESCs and assisted with embryo manipulation. XY and YL performed some of the experiments. YA contributed to histological analysis and sequencing. YS and QZ designed the project, performed some of the experiments, analyzed the data, and wrote the manuscript. All authors contributed to the article and approved the submitted version. 


\section{FUNDING}

This work was supported in part by the Japan Society for the Promotion of Science (KAKENHI; grant nos. 18K15921 and $18 \mathrm{H} 02817 \mathrm{G})$.

\section{ACKNOWLEDGMENTS}

We thank Takenori Sakuma, Nae Saito, and Sumika Uchiyama for assistance with embryo manipulation, mouse

\section{REFERENCES}

1. Arauchi A, Shimizu T, Yamato M, Obara T, Okano T. Tissue-engineered thyroid cell sheet rescued hypothyroidism in rat models after receiving total thyroidectomy comparing with nontransplantation models. Tissue Eng Part A (2009) 15(12):3943-9. doi: 10.1089/ten.tea.2009.0119

2. Yang Y, Opara EC, Liu Y, Atala A, Zhao W. Microencapsulation of porcine thyroid cell organoids within a polymer microcapsule construct. Exp Biol Med (Maywood) (2017) 242(3):286-96. doi: 10.1177/1535370216673746

3. Clarke N, Kabadi UM. Optimizing treatment of hypothyroidism. Treat Endocrinol (2004) 3:217-21. doi: 10.2165/00024677-200403040-00003

4. Arauchi A, Matsuura K, Shimizu T, Okano T. Functional Thyroid Follicular Cells Differentiation from Human-Induced Pluripotent Stem Cells in Suspension Culture. Front Endocrinol (Lausanne) (2017) 8:103. doi: 10.3389/ fendo.2017.00103

5. Huang Y, Yamanouchi K, Sakai Y, Kuba S, Sakimura C, Morita M, et al. Fabrication of Functional Cell Sheets with Human Thyrocytes from NonTumorous Thyroid Tissue. Tissue Eng Regener Med (2019) 16(5):491-9. doi: 10.1007/s13770-019-00198-8

6. Arufe MC, Lu M, Kubo A, Keller G, Davies TF, Lin RY. Directed differentiation of mouse embryonic stem cells into thyroid follicular cells. Endocrinology (2006) 147:3007-15. doi: 10.1210/en.2005-1239

7. Arufe MC, Lu M, Lin RY. Differentiation of murine embryonic stem cells to thyrocytes requires insulin and insulin-like growth factor-1. Biochem Biophys Res Commun (2009) 381:264-70. doi: 10.1016/j.bbrc.2009.02.035

8. Ma R, Latif R, Davies TF. Thyrotropin-independent induction of thyroid endoderm from embryonic stem cells by activin A. Endocrinology (2009) 150:1970-5. doi: 10.1210/en.2008-1374

9. Jiang N, Hu Y, Liu X, Wu Y, Zhang H, Chen G, et al. Differentiation of E14 mouse embryonic stem cells into thyrocytes in vitro. Thyroid (2010) 20:77-84. doi: 10.1089/thy.2008.0291

10. Longmire TA, Ikonomou L, Hawkins F, Christodoulou C, Cao YX, Kwok LW, et al. Efficient derivation of purified lung and thyroid progenitors from embryonic stem cells. Cell Stem Cell (2012) 10:398-411. doi: 10.1016/ j.stem.2012.01.019

11. Kurmann AA, Serra M, Hawkins F, Rankin SA, Mori M, Astapova I, et al. Regeneration of Thyroid Function by Transplantation of Differentiated Pluripotent Stem Cells. Cell Stem Cell (2015) 17(5):527-42. doi: 10.1016/ j.stem.2015.09.004

12. Antonica F, Kasprzyk DF, Opitz R, Iacovino M, Liao XH, Dumitrescu AM, et al. Generation of functional thyroid from embryonic stem cells. Nature (2012) 491:66-71. doi: 10.1038/nature11525

13. Ma R, Latif R, Davies TF. Thyroid follicle formation and thyroglobulin expression in multipotent endodermal stem cells. Thyroid (2013) 23:385-91. doi: 10.1089/thy.2012.0644

14. Ma R, Latif R, Davies TF. Human embryonic stem cells form functional thyroid follicles. Thyroid (2015) 25(4):455-61. doi: 10.1089/thy.2014.0537

15. Dame K, Cincotta S, Lang AH, Sanghrajka RM, Zhang L, Choi J, et al. Thyroid Progenitors Are Robustly Derived from Embryonic Stem Cells through Transient, Developmental Stage-Specific Overexpression of Nkx2-1. Stem Cell Rep (2017) 8(2):216-25. doi: 10.1016/j.stemcr.2016.12.024

16. Kobayashi T, Yamaguchi T, Hamanaka S, Kato-Itoh M, Yamazaki Y, Ibata M, et al. Generation of rat pancreas in mouse by interspecific blastocyst injection housing, and mouse tissue sampling. We thank Rie Natsume for assistance with embryo micro-injection, and Shinichi Kenmotsu and Hayato Ohshima for assistance with micro-CT.

\section{SUPPLEMENTARY MATERIAL}

The Supplementary Material for this article can be found online at: https://www.frontiersin.org/articles/10.3389/fendo.2020. 609697/full\#supplementary-material

of pluripotent stem cells. Cell (2010) 142:787-99. doi: 10.1016 j.cell.2010.07.039

17. Yamaguchi T, Sato H, Kato-Itoh M, Goto T, Hara H, Sanbo M, et al. Interspecies organogenesis generates autologous functional islets. Nature (2017) 542:191-6. doi: 10.1038/nature21070

18. Usui J, Kobayashi T, Yamaguchi T, Knisely AS, Nishinakamura R, Nakauchi H, et al. Generation of kidney from pluripotent stem cells via blastocyst complementation. Am J Pathol (2012) 180:2417-26. doi: 10.1016/j.ajpath. 2012.03.007

19. Teppei G, Hiromasa H, Makoto S, Masaki H, Sato H, Yamaguchi T, et al. Generation of pluripotent stem cell-derived mouse kidneys in Sall1-targeted anephric rats. Nat Commun (2019) 10(Article number):451. doi: 10.1038/s41467-019-08394-9

20. Wang X, Shi H, Zhou J, Zou Q, Zhang Q, Gou S, et al. Generation of rat blood vasculature and hematopoietic cells in rat-mouse chimeras by blastocyst complementation. J Genet Genomics May (2020) 19:S1673-8527(20)30086-2. doi: 10.1016/j.jgg.2020.05.002

21. Mori M, Furuhashi K, Danielesson JA, Hirata Y, Kakiuchi M, Lin CS, et al. Generation of functional lungs via conditional blastocyst complementation using pluripotent stem cells. Nat Med (2019) 25:1691-8. doi: 10.1038/s41591019-0635-8

22. Kitahara A, Ran Q, Oda K, Yasue A, Abe M, Ye X, et al. Generation of Lungs by Blastocyst Complementation in Apneumic Fgf10-Deficient Mice. Cell Rep (2020) 31(6):107626. doi: 10.1016/j.celrep.2020.107626

23. Sekine K, Ohuchi H, Fujiwara M, Yamasaki M, Yoshizawa T, Sato T, et al. Fgf10 is essential for limb and lung formation. Nat Genet (1999) 21:138-41. doi: $10.1038 / 5096$

24. Ohuchi H, Hori Y, Yamasaki M, Harada H, Sekine K, Kato S, et al. FGF10 acts as a major ligand for FGF receptor 2 IIIb in mouse multi-organ development. Biochem Biophys Res Commun (2000) 277:643-9. doi: 10.1006/bbrc.2000.3721

25. Yasue A, Mitsui S, Watanabe T, Sakuma T, Oyadomari S, Yamamoto T, et al. Highly efficient targeted mutagenesis in one-cell mouse embryos mediated by the TALEN and CRISPR/Cas systems. Sci Rep (2014) 4:5705. doi: 10.1038/srep05705

26. Bellusci S, Grindley J, Emoto H, Itoh N, Hogan BL. Fibroblast growth factor 10 (FGF10) and branching morphogenesis in the embryonic mouse lung. Development (1997) 124(23):4867-78.

27. Abler LL, Mansour SL, Sun X. Conditional gene inactivation reveals roles for Fgf10 and Fgfr2 in establishing a normal pattern of epithelial branching in the mouse lung. Dev Dyn (2009) 238(8):1999-2013. doi: 10.1002/dvdy.22032

28. Yuan T, Volckaert T, Chanda D, Thannickal VJ, De Langhe SP. Fgf10 signaling in lung development, homeostasis, disease, and repair after injury. Front Genet (2018) 9:418. doi: 10.3389/fgene.2018.00418

29. Revest JM, Spencer-Dene B, Kerr K, De Moerlooze L, Rosewell I, Dickson C. Fibroblast growth factor receptor 2-IIIb acts upstream of Shh and Fgf4 and is required for limb bud maintenance but not for the induction of Fgf8, Fgf10, Msx1, or Bmp4. Dev Biol (2001) 231:47-62. doi: 10.1006/dbio.2000.0144

30. Liang S, Johansson E, Barila G, Altschuler DL, Fagman H, Nilsson M. A branching morphogenesis program governs embryonic growth of the thyroid gland. Development (2018) 145(2):dev146829. doi: 10.1242/dev.146829

31. Teshima TH, Lourenco SV, Tucker AS. Multiple cranial organ defects after conditionally knocking out Fgf10 in the neural crest. Front Physiol (2016) 7:488. doi: $10.3389 /$ fphys.2016.00488

32. Parlato R, Rosica A, Rodriguez-Mallon A, Affuso A, Postiglione MP, Arra C, et al. An integrated regulatory network controlling survival and migration in 
thyroid organogenesis. Dev Biol (2004) 276:464-75. doi: 10.1016/ j.ydbio.2004.08.048

33. Fagman H, Nilsson M. Morphogenetics of early thyroid development. J Mol Endocrinol (2011) 46(1):R33-42. doi: 10.1677/JME-10-0084

34. Nilsson M, Fagman H. Development of the thyroid gland. Development (2017) 144:2123-40. doi: 10.1242/dev.145615

35. Itoh N. Fgf10: a multifunctional mesenchymal-epithelial signaling growth factor in development, health, and disease. Cytokine Growth Factor Rev (2016) 28:63-9. doi: 10.1016/j.cytogfr.2015.10.001

36. Hollenberg AN, Choi J, Serra M, Kotton DN. Regenerative therapy for hypothyroidism: Mechanisms and possibilities. Mol Cell Endocrinol (2017) 445:35-41. doi: 10.1016/j.mce.2016.11.012

37. Wen B, Li E, Ustiyan V, Wang G, Guo M, Na CL, et al. In Vivo Generation of Lung and Thyroid Tissues from Embryonic Stem Cells using Blastocyst
Complementation. Am J Respir Crit Care Med (2020) 2. doi: 10.1164/ rccm.201909-1836OC

Conflict of Interest: The authors declare that the research was conducted in the absence of any commercial or financial relationships that could be construed as a potential conflict of interest.

Copyright (c) 2020 Ran, Zhou, Oda, Yasue, Abe, Ye, Li, Sasaoka, Sakimura, Ajioka and Saijo. This is an open-access article distributed under the terms of the Creative Commons Attribution License (CC BY). The use, distribution or reproduction in other forums is permitted, provided the original author(s) and the copyright owner(s) are credited and that the original publication in this journal is cited, in accordance with accepted academic practice. No use, distribution or reproduction is permitted which does not comply with these terms. 